\title{
Spatio-Temporal Prior Shape Constraint for Level Set Segmentation ${ }^{\star}$
}

\author{
T. Bailloeul ${ }^{1,2,3}$, V. Prinet ${ }^{1}$, B. Serra ${ }^{2}$, and P. Marthon ${ }^{3}$ \\ 1 LIAMA, Institute of Automation, Chinese Academy of Sciences, \\ P.O Box 2728, Beijing 100080, China \\ Phone: (+86 10) 82614462 / Fax : (+86 10) 62647458 \\ tbailloeul@liama.ia.ac.cn, prinet@nlpr.ia.ac.cn \\ 2 Alcatel Space, 100 bd du Midi, 06156 Cannes La Bocca, France \\ Phone: (+33)492926726 / Fax: $(+33) 492927660$ \\ Bruno.Serraespace.alcatel.fr \\ 3 LIMA (IRIT), 2 rue Camichel, 31071 Toulouse, France \\ Phone/Fax: $(+33) 561588353$ \\ Philippe.Marthon@enseeiht.fr
}

\begin{abstract}
This paper exposes a novel formulation of prior shape constraint incorporation for the level set segmentation of objects from corrupted images. Applicable to variational frameworks, the proposed scheme consists in weighting the prior shape constraint by a function of time and space to overcome local minima issues of the energy functional. Pose parameters which make the prior shape constraint invariant from global transformations are estimated by the downhill simplex algorithm, which is more tractable and robust than the traditional gradient descent. The proposed scheme is simple, easy to implement and can be generalized to any variational approach incorporating a single prior shape. Results illustrated with different kinds of images demonstrate the efficiency of the method.
\end{abstract}

\section{Introduction}

Since the early nineties the incorporation of a priori and geometric high level information within active contours frameworks has become popular to segment objects from physically corrupted images. A constraint applied on the shape of a free form deformable model is a common approach to address the issue of object segmentation from altered data. Unlike generic geometric constraints such as contour regularization, a prior shape constraint is specific and derived from extrinsic information. It aims at restricting the space of possible shapes embodied by a segmenting curve and therefore enables to overcome image artifacts which influence is penalized by the shape prior constraint. The Medical Imaging

* This project is supported by the Chinese MOST 863 programme and Alcatel Space. We thank Wanlin Zhu for providing the medical image used in our experiments and Ian Jermyn for the discussions we had about the proposed approach. We thank the anonymous reviewers for their remarks and suggestions.

A. Rangarajan et al. (Eds.): EMMCVPR 2005, LNCS 3757, pp. 503-519 2005.

(C) Springer-Verlag Berlin Heidelberg 2005 
community was the first to use shape constrained deformable models as it has to deal with images frequently distorted by noise, occlusions and poor contrast of organs to be segmented [1,2, 3, 4, The application of these model-based schemes was later extended to manufactured and natural scenes objects as well as object tracking from video sequences $[5,6,7,8,9,10,11$.

The prior shape constraint can be derived from a single or a collection of reference shapes. In the latter case statistical frameworks take advantage of the geometric variability across the different instances of the object, which conveys a larger although restricted space of possible shapes of the active contour. As a result, the segmenting curve has a globally constrained shape while being able to include local disparities contained in the training samples. Shape samples are first aligned to remove pose differences which could bias the retrieval of intrinsic shape variabilities from the database. Then a statistical prior shape model is built to determine the variability across the family of shapes. Finally the shape model is integrated in the segmentation model: the constraint could be naturally incorporated in the active contour evolution equation, exerted in the training shapes space or inserted extrinsically as a corrective term. The shape constraint integration is often invariant from a global transformation which parameters need to be estimated as the segmenting curve evolves. In [1] shapes are represented by their projection on an elliptic Fourier basis. Prior probability distributions are calculated on the representation parameters from the training samples and are used in a maximum a posteriori (MAP) framework to constrain the segmenting curve that captures image high gradients. In 2, 5, 3, 12] shape samples are projected in an orthogonal subspace by principal component analysis (PCA), which retains the main variations modes from the different instances. Differences arise among these schemes towards the representation of the shapes as well as the way the shape constraint is applied. In [2] the active contour is explicitly represented as a snake [13, and in [5] shapes are projected on a B-spline basis. The main limitation of the aforementioned works is their inability to make the evolving contour undergo topological changes which are naturally handled by the distance level set representation of curves [14,15]. In [3,12] shapes are implicitly represented by their level set functions. In 2, 12, the shape constraint is directly applied in the PCA subspace whereas it is achieved by a corrective term to the evolution equation in a MAP approach in [3]. In [5] the shape constraint incorporation is achieved through the input in an energy functional of a Mahalanobis distance measuring the discrepancy between the evolving curve and the statistical prior shape model. In 6] Paragios and Rousson propose a nonstationary pixel-wise Gaussian model which fully takes advantage of the level set representation of shapes and preserves the ability to capture local deformations.

In case the different instances of the object reduce to a sole shape prior, the training shapes space contracts to a singleton set and statistical schemes for prior shape modeling are no longer valid. As a consequence shape prior incorporation is achieved in a harder fashion as local variabilities of the contour from the reference shape are only governed by the balance between image and prior shape information. Incorporation of the shape prior can be achieved through the 
formulation of a distance measuring the dissimilarity between the segmenting curve and the reference shape, modulo a global transformation. In [4] shape constrained geodesic active contours are used to retrieve boundaries of corrupted objects from medical images. In this variational framework the shape distance is formalized as the sum of the squared distances between the evolving contour points and the shape prior. Unlike this edge-to-edge dissimilarity measure, surface terms comparing the active contour and the shape prior were proposed for level set segmentation $9,10,8$. The dissimilarity energy can be either the squared difference between the level set functions embedding the contour and the shape prior [9, or the difference of areas contained by the contour and the reference shape 8 . In the latter case, the shape energy does not depend on the image domain $\Omega$. Further works towards shape energy independent from $\Omega$ and the extension to shape prior with multiple components can be found in [10] with the formulation of a symmetric pseudo-distance. In 7 . Foulonneau et al. formulate a distance between high order geometric moments of the evolving curve and the shape prior to apply the constraint. The moments are projected on a Legendre polynomial basis to decrease the redundancy of the shape representation.

In this paper we argue about the sensitivity to local minima of shape constrained level segmentation in variational frameworks. We propose to address the issue of the balance between shape and image information responsible for local minima occurrence. We tackle this problem while considering a segmenting curve constrained by a single shape prior. The next section states and details further the problem we aim at resolving. The present paper formalizes and extends the work undertaken in [16] and applied to remote sensing imagery.

\section{Problem Statement}

Assuming the evolving active contour is implicitly represented by a signed Euclidean distance level set function $\phi$ [14, 15, the shape prior incorporation in variational frameworks can be generally formalized as follows:

$$
J(\phi, \psi)=J_{\text {image }}(\phi)+\lambda J_{\text {shape }}(\phi, \psi)
$$

where $J$ is the energy functional, $J_{\text {image }}$ is the image-based energy which aims at driving the segmenting curve to the object boundaries and $J_{\text {shape }}$ is the shape constraint energy that restricts possible shapes embodied by the contour. $J_{\text {shape }}$ measures the dissimilarity between the evolving contour and a shape reference $\psi$. The higher the discrepancy, the higher $J_{\text {shape }}$ :

$$
J_{\text {shape }}(\phi, \psi)=\int_{\Omega} \mathcal{D}(\phi(\mathbf{x}), \psi(\mathbf{x})) d \mathbf{x}
$$

where $\mathcal{D}$ is a function measuring the distance over the image domain $\Omega$ between the active contour embedded in $\phi$ and the shape prior represented by the level set function $\psi$. The constant $\lambda \in \mathbb{R}^{+}$balances the influence of the shape constraint 
with respect to the image information. A first issue referring to the value of the constant $\lambda$ arises from equation (11). On the one hand, a too low value of $\lambda$ will not enable to overcome image artifacts, i.e. the local minima of $J$ corresponding to wrong segmentations will be numerous (in the rest of the paper these minima will be denoted as local minima of the first kind). On the other hand, a high value of $\lambda$ will restrict too much the freedom of movement of the contour, thus drastically limiting the ways it can escape from local minima (second kind). To find an optimal value for $\lambda$, which could fairly balance shape and image information is not trivial since this optimum may depend on the image to be analyzed as well as the type and level of corruption of image data, which are not always known in advance. The tuning of the constant $\lambda$ is a difficult task which is even more intractable when the target object to be retrieved in the image is surrounded by peripheral objects with similar statistical properties or when it is poorly discriminated from the background. Indeed in such case image information which drives the contour to the object boundaries is weakened regarding to the shape constraint which therefore becomes predominant. The shape information is then over-weighted, which leads to the second aforementioned local minima problem. This unwelcome effect is even more severe when the segmenting curve initialization is distant from the target as the convergence to the right solution become hazardous. The issue of local minima inherent to the unsuitable balance between the shape constraint and the image information is addressed in this paper. To alleviate this problem we propose to embed the shape prior in a soften fashion while spatially and temporally relaxing and reinforcing the shape constraint during the convergence process. Such flexible incorporation that we will describe in section three conveys a better control of the degrees of freedom of the segmenting curve to overcome local minima obstacles.

The distance $\mathcal{D}$ in (2) is made invariant from a global transformation which best maps the prior shape to the evolving active contour:

$$
\psi=\psi_{0} \circ T_{\xi}
$$

where $T$ is a global transformation which parameters are $\xi=\left\{\xi_{1}, \ldots, \xi_{n}\right\} ; \psi_{0}$ is the static shape prior level set. Invariance from global transformation is achieved while finding the optimal parameters $\xi_{\text {opt }}$ which minimize $J_{\text {shape }}$ :

$$
\xi_{\text {opt }}=\underset{\xi}{\arg \min } J_{\text {shape }}\left(\phi, \psi_{0} \circ T_{\xi}\right)
$$

The second problem we address in this paper relates to the estimation of the optimum parameters $\xi_{\text {opt }}$ which minimize $J_{\text {shape }}$. According to the expression of $J_{\text {shape }}$ it may not always be possible to analytically deduce $\xi_{\text {opt }}$ from:

$$
\left(\frac{\partial J_{\text {shape }}}{\partial \xi_{i}}\right)_{i=1, \ldots, n}=0
$$

As a consequence numerical schemes have to be used to retrieve $\xi_{\text {opt }}$. A commonly adopted method is a gradient descent scheme which sequentially estimates $\xi_{i}$ by 
$n$ iterative descents [2, 17, 12, 4, 6, 8, Practical implementation of such scheme is challenging as a wrong estimation in the $i^{\text {th }}$ descent may bias the estimation of the $n-1$ remaining parameters, preventing in the end to retrieve $T$. Cremers et. al touched on the problem of tuning such gradient-descent scheme for parameters optimization in 11. They proposed an alternative way avoiding numerical optimization: invariance from translation and isotropic scaling is achieved in projecting the level set functions $\phi$ and $\psi$ in their intrinsic reference systems. In his approach the scale and translation parameters are not derived from (5) but are equal to the first and second order moments of the shape. Invariance from rotation with such scheme is more challenging and not achieved yet. In this article we propose an alternative to the gradient descent scheme for pose parameters estimation during the segmentation process. The section four details our alternative based on the downhill simplex optimization method, which is parameter free and estimates the pose parameters simultaneously and more robustly. The combination of the soften shape constraint with the simplex pose parameters estimation yields some promising experimental results that are showed in section five with different kinds of images. We compare our proposed scheme with constant and simpler spatio-temporal prior shape incorporations. The performances difference between the simplex and gradient descent schemes is also investigated. Finally we discuss the proposed approach and the results illustrated in this paper and conclude in section six.

\section{Spatio-Temporal Shape Constraint Weight}

To address the practical problem of local minima, we first turn the shape prior weight $\lambda$ in equation (11) into a function of space. It is meant to relax the shape prior influence within a restricted area close to the 0-level set of the reference template $\psi$ while preserving the original uniform shape constraint far from it. Such spatially restricted relaxation will convey a limited freedom to the evolving active contour which shape space is therefore enlarged. Consequently, the contour may globally keep the reference template shape while allowing local variations, which may be a desirable property to avoid local minima of the second kind. We propose to formalize this spatial relaxation by a symmetric function of the distance to the shape prior:

$$
\lambda(\psi(\mathbf{x}))=1-e^{-\left(\frac{\psi(\mathbf{x})}{d}\right)^{2}}
$$

This one-parameter function has a minimum value at the 0-level set of the prior template while it increases and asymptotically tends to a constant farther. $d$ is the parameter controlling the size of the area where the prior shape is spatially relaxed. Simpler or more intuitive formulations than (6) such as piecewise linear functions of $\psi(\mathbf{x})$ would fulfill the same relaxation requirements. However as we may empirically demonstrate and discuss further in section 5.3, the derivability and the presence of a stationary point at $\psi(\mathbf{x})=0$ is a property which improves the present method. 
The existence of the freedom space is intended to prevent the segmenting curve from being trapped by local minima of the second kind, nevertheless it would not enable to deal with corrupted images as allowed local variations may correspond to artifacts we aim at overcoming (local minima of the first kind). We propose to temporally decrease the freedom space to $\{\emptyset\}$ in the iterative convergence process starting from time $t_{1}$. As the freedom space narrows the active contour shape space decreases, which augments the shape constraint efficiency uniformly all over the image domain. The underlying idea is to reach a rough segmentation while the prior shape constraint is spatially relaxed $\left(t<t_{1}\right)$ and to enhance the shape penalty in a second time to overcome image alterations $\left(t \geq t_{1}\right)$. As a result, the prior shape weight function depends on space and on time as well. The decrease of the freedom space is to be achieved in turning the parameter $d$ into a decreasing linear function of time:

$$
d(t)=\left\{\begin{array}{cc}
d_{0} & \text { if } t<t_{1} \\
\left(\varepsilon-d_{0}\right) \frac{t-t_{1}}{t_{2}-t_{1}}+d_{0} & \text { if } t_{1} \leq t<t_{2} \\
\varepsilon & \text { if } t_{2} \leq t
\end{array}\right.
$$

where $\left(d_{0}, \varepsilon\right) \in \mathbb{R}^{*+}$ with $d_{0}>\varepsilon$ and $\varepsilon \ll 1$.

Finally, we enhance prior shape relaxation and reinforcement in multiplying (6) by an increasing function $\lambda_{a}(t)$ of the convergence time $t$ and which will globally rule the amplitude of the prior shape weight function. At the beginning of the iterative process, the amplitude of the prior shape weight is low to convey more flexibility to the evolving active contour. Starting from $t_{1}$, the amplitude increases to reach a maximum value which supports the shape constraint and enables the segmentation of corrupted objects in the image. In the end, the spatio-temporal prior shape weight function $\lambda: \mathbb{R} \times \mathbb{R} \rightarrow \mathbb{R}^{+}$can be formalized as follows:

$$
\lambda(t, \psi(\mathbf{x}))=\lambda_{a}(t) \lambda_{\text {space }}(\psi(\mathbf{x}))
$$

where $\lambda_{a}(t)$ is ranging from $\lambda_{\min }$ and $\lambda_{\max }$ and is akin to the opposite function of (17) and $\lambda_{\text {space }}(\psi(\mathbf{x}))=1-e^{-\left(\frac{\psi(\mathbf{x})}{d(t)}\right)^{2}}$. The shape energy is then reformulated as:

$$
J_{\text {shape }}(\phi, \psi, t)=\int_{\Omega} \lambda(t, \psi(\mathbf{x})) \mathcal{D}(\phi(\mathbf{x}), \psi(\mathbf{x})) d \mathbf{x}
$$

A spatio-temporal variation of the shape prior constraint has already been carried out in [9] for a different purpose. The authors introduced a labelling function which defines where the shape constraint is applied and which evolves in space and time. As a result the segmentation scheme is able to segment familiar and corrupted objects as well as unfamiliar ones. This method was extended in 8 to similarity transformation invariance and with a different formulation of the dynamic labelling function. Satisfying results are showed for segmenting object lacking discrimination from the background, which is the point we also address here. However our approach does not make use of extrinsic labelling function, which makes our scheme simpler and more self-consistent. The figure 1 


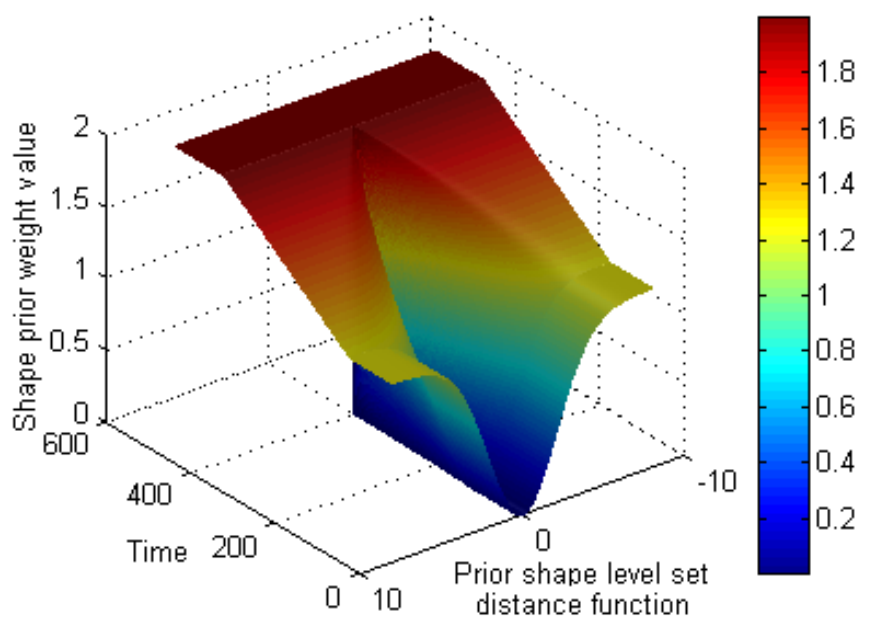

Fig. 1. Spatio-temporal shape prior weight function with $d_{0}=3, t_{1}=100, t_{2}=400$, $\lambda_{\min }=1.25$ and $\lambda_{\max }=2$

illustrates the spatio-temporal profile in three dimensions. Two questions arise from the definition of the proposed spatio-temporal shape constraint. The first deals with the choice of $t_{1}$ which should depend on the image features. As we already mentioned, a solution would be to reach a rough segmentation with a low and spatially constrained shape prior and subsequently increase the shape constraint efficiency. In that case $t_{1}$ is the time needed to reach the convergence of the coarse segmentation. The second issue applies to the influence on pose parameters estimation of discrepancies between the contour and the shape prior. Since variations from the reference template are allowed within the freedom space, it might be crucial to robustly retrieve pose parameters in spite of local discrepancies. We address the latter issue in the next section.

\section{Shape Prior Invariance: A Simplex Optimization Scheme for Parameters Estimation}

The sequential estimation of pose parameters by gradient descent as well as the tuning of the time step for each descent make the use of this scheme unsuitable. As the descents estimate parameters with different geometric meanings (e.g. rotation, scaling, translation, etc...), it is not clear how fast should one descent be with respect to the others. Since the $n$ gradient descents are dependent, a slightly wrong parameter estimation in one descent might impact the $n-1$ remaining ones to finally make the pose parameters retrieval diverge. We propose to use the downhill simplex method [18 to solve the optimization problem stated in equation (44). The simplex method is an efficient zero-order iterative scheme designed to minimize non-linear cost functions. Unlike the gradient descent technique, the 
simplex does not use derivatives and it estimates robustly the optimal parameters. A simplex is a $n$-dimensional convex polyhedron defined in the parameters space by $n+1$ vertices which evaluate the cost function with different sets of parameters $\xi$. Initially, the first vertex of the simplex is derived from some given initial parameters $\xi_{0}$ and the rest of the polyhedron is built in the same way while adding an individual variation $\delta_{i}$ to each component of $\xi_{0}$. The subsequent iterative algorithm is as follows:

1. The simplex vertices are sorted according to their corresponding cost function values. Let be $\mathbf{b}$ the best vertex among them (minimum cost function value), $\mathbf{w}$ the worst and $\mathbf{a}$ the best after $\mathbf{b}$. As the method intends to find a minimum of the cost function, it attempts to build a new simplex far from the worst vertex $\mathbf{w}$. To do so, one tries to replace $\mathbf{w}$ by a vertex $\mathbf{c}$ better than $\mathbf{b}$ :

2. A better candidate $\mathbf{c}$ is tested as the reflexion of $\mathbf{w}$ with respect to the hyperplane $\mathcal{H}$ opposite to $\mathbf{w}$ (reflexion of the simplex). If such $\mathbf{c}$ is better than b it is possible to look farther in this direction (expansion of the simplex). The best candidate among the reflexion and expansion results is chosen to replace $\mathbf{w}$.

3. If none of the previous attempts is better than $\mathbf{b}$, one tries two candidates $\mathbf{c}_{1}$ and $\mathbf{c}_{2}$ laying on each side of $\mathcal{H}$ and belonging to the hyper-line normal to $\mathcal{H}$ which goes through $\mathbf{w}$. The best candidate will replace $\mathbf{w}$ if it is better than a (contraction of the simplex).

4. If neither $\mathbf{c}_{\boldsymbol{1}}$ nor $\mathbf{c}_{\boldsymbol{2}}$ is better than $\mathbf{a}$, it means $\mathbf{b}$ is already close to the expected solution. In that case the best vertex $\mathbf{b}$ is kept and each remaining vertex is replaced by the middle point of the segment connecting it to $\mathbf{b}$.

5. Once the new simplex is built, the algorithm loops from steps 1 to 5 until the score difference between two consecutive best vertices is below a given threshold $\varepsilon_{\text {simplex }}$. The estimated optimal parameters $\hat{\xi}_{\text {opt }}$ are the ones corresponding to the last best vertex $\mathbf{b}$.

The main advantage of the simplex algorithm over the gradient descent scheme is the simultaneous estimation of the parameters. In that way the uneasy and intricate tuning of the $n$ dependent gradient descents is avoided. Besides, the simplex is known to estimate more robustly parameters which values are far from $\xi_{0}$. Indeed the simplex does not need to follow gradient descent curves in the cost function space, which makes it less sensitive to local minima. However one limitation of the simplex method is its computational cost as it needs numerous evaluations of the cost function to be minimized. We will see in section 5.2 how to decrease the computational load for evaluating the cost function by using the narrow band technique. A too high number of parameters to be estimated might also increase the complexity of the simplex method. However in two dimensions, affine and projective transformations have less than ten parameters, which is manageable by the simplex. Finally, the performances of the simplex algorithm also depend on the size of the initial simplex as well as the value of $\varepsilon_{\text {simplex }}$ : a large initial simplex might enable to retrieve optimal parameters far from $\xi_{0}$ 
at the cost of a higher computational load; a smaller $\varepsilon_{\text {simplex }}$ would yield finer estimates $\hat{\xi}_{\text {opt }}$ with longer convergence times. In the experiments detailed in the next section, we chose to estimate pose parameters after each evolution of the active contour and with $\varepsilon_{\text {simplex }}=10^{-4}$.

\section{Experiments}

\subsection{Image-Based and Shape Constraint Models}

In our experiments we make use of the region-based formulation of the Bayesian MAP deformable model formerly proposed in [19] in order to drive the active contour to the target object in the image. This approach best befits segmentation of piecewise smooth components of an image $I$ :

$$
\begin{gathered}
J_{\text {image }}(\phi)=\int_{\Omega}\left(\frac{\left(I(\mathbf{x})-\bar{I}_{\text {in }}(\phi(\mathbf{x}, t))\right)^{2}}{2 \sigma_{\text {in }}^{2}(\phi(\mathbf{x}, t))}+\ln \sqrt{2 \pi \sigma_{\text {in }}^{2}(\phi(\mathbf{x}, t))}\right) H_{a}(\phi(\mathbf{x}, t)) d \mathbf{x} \\
+\int_{\Omega}\left(\frac{\left(I(\mathbf{x})-\bar{I}_{\text {out }}(\phi(\mathbf{x}, t))\right)^{2}}{2 \sigma_{\text {out }}^{2}(\phi(\mathbf{x}, t))}+\ln \sqrt{2 \pi \sigma_{\text {out }}^{2}(\phi(\mathbf{x}, t))}\right)\left(1-H_{a}(\phi(\mathbf{x}, t))\right) d \mathbf{x}
\end{gathered}
$$

where $\bar{I}$ and $\sigma^{2}$ respectively denote the image mean and variance grey level. Subscripts in and out refer to the computation of these statistical quantities inside and outside the evolving active contour:

$$
\begin{gathered}
\bar{I}_{\text {in }}(\phi(\mathbf{x}, t))=\frac{\int_{\Omega} I(\mathbf{x}) H_{a}(\phi(\mathbf{x}, t)) d \mathbf{x}}{\int_{\Omega} H_{a}(\phi(\mathbf{x}, t)) d \mathbf{x}} \\
\bar{I}_{\text {out }}(\phi(\mathbf{x}, t))=\frac{\int_{\Omega} I(\mathbf{x})\left(1-H_{a}(\phi(\mathbf{x}, t))\right) d \mathbf{x}}{\int_{\Omega}\left(1-H_{a}(\phi(\mathbf{x}, t))\right) d \mathbf{x}} \\
\sigma_{\text {in }}^{2}(\phi(\mathbf{x}, t))=\frac{\int_{\Omega}\left(I(\mathbf{x})-\bar{I}_{\text {in }}(\phi(\mathbf{x}, t))\right)^{2} H_{a}(\phi(\mathbf{x}, t)) d \mathbf{x}}{\int_{\Omega} H_{a}(\phi(\mathbf{x}, t)) d \mathbf{x}} \\
\sigma_{\text {out }}^{2}(\phi(\mathbf{x}, t))=\frac{\int_{\Omega}\left(I(\mathbf{x})-\bar{I}_{\text {out }}(\phi(\mathbf{x}, t))\right)^{2}\left(1-H_{a}(\phi(\mathbf{x}, t))\right) d \mathbf{x}}{\int_{\Omega}\left(1-H_{a}(\phi(\mathbf{x}, t))\right) d \mathbf{x}}
\end{gathered}
$$

The active contour is embedded as a level set function $\phi$, which is assumed to be positive inside the contour. $H_{a}$ represents a regularized approximation of the Heaviside function. Edge-based and contour regularization terms have been intentionally omitted in (10) since we only investigate region-based active contours. Besides, the shape constraint to be introduced will act as a contour regularizer. We chose the shape constraint energy independent from the domain $\Omega$ and proposed in [8]. It measures the discrepancy between areas included in the evolving active contour and the shape prior:

$$
\mathcal{D}(\phi(\mathbf{x}), \psi(\mathbf{x}))=\left(H_{a}(\phi(\mathbf{x}))-H_{a}(\psi(\mathbf{x}))\right)^{2}
$$


The gradient of the overall energy functional $J$ with respect to the level set function $\phi$ yields the following active contour evolution equation:

$$
\begin{gathered}
\phi(\mathbf{x}, t)_{t}=\delta_{a}(\phi(\mathbf{x}, t))\left\{-\frac{\left(I(\mathbf{x})-\bar{I}_{\text {in }}(\phi(\mathbf{x}, t))\right)^{2}}{2 \sigma_{\text {in }}^{2}(\phi(\mathbf{x}, t))}+\frac{\left(I(\mathbf{x})-\bar{I}_{\text {out }}(\phi(\mathbf{x}, t))\right)^{2}}{2 \sigma_{\text {out }}^{2}(\phi(\mathbf{x}, t))}\right. \\
\left.+\ln \left(\frac{\sigma_{\text {out }}^{2}(\phi(\mathbf{x}, t))}{\sigma_{\text {in }}^{2}(\phi(\mathbf{x}, t))}\right)+2 \lambda(t, \psi(\mathbf{x}))\left[H_{a}(\phi(\mathbf{x}, t))-H_{a}(\psi(\mathbf{x}, t))\right]\right\}
\end{gathered}
$$

\subsection{Invariance from Similarity Transformation and Algorithm}

In these experiments we propose to consider invariance from a similarity transformation which includes isotropic scaling, rotation and translation:

$$
T \mathbf{x}=s\left[\begin{array}{cc}
\cos \theta & -\sin \theta \\
\sin \theta & \cos \theta
\end{array}\right] \mathbf{x}+\left[\begin{array}{l}
\mu_{x} \\
\mu_{y}
\end{array}\right]
$$

with the scaling factor noted as $s\left(s \in \mathbb{R}^{*+}\right), \theta$ refers to the rotation angle and $\mu$ to the translation. As demonstrated in [20, the relationship between a level set function and its transformed by similarity is solved analytically, which eases the computation of $\psi(\mathbf{x}, t)$. We use the narrow banding technique to decrease the computational cost of our algorithm. The reason for using such technique is twofold. First it decreases the cost for evolving the active contour and redistancing the level set function $\phi$. Second, it can also decrease the computational cost of the simplex-based pose parameters estimation. Indeed the evaluation of the cost function $J_{\text {shape }}$ using equation (9), which is needed to build the simplex vertices, can be reduced by computing shape discrepancies within a narrow band around the 0-level set of $\phi$ :

$$
J_{\text {shape }}(\phi, \psi)=\int_{\Omega} N\left(\phi(\mathbf{x}), \varepsilon_{b}\right) \lambda(t, \psi(\mathbf{x})) \mathcal{D}(\phi(\mathbf{x}), \psi(\mathbf{x})) d \mathbf{x}
$$

where the function $N\left(\phi(\mathbf{x}), \varepsilon_{b}\right)$ is equal to 1 if $|\phi(\mathbf{x})| \leq \varepsilon_{b}$, otherwise it is equal to 0 . The band half size $\varepsilon_{b}$ is then a compromise between computational efficiency and the pose parameters accuracy: a too narrow band will not yield a discriminative measure of discrepancy, which may lead the simplex optimization to erroneous results. A systematic re-initialization of the level set function $\phi$ is also needed to provide a good measure of dissimilarity with the shape prior and therefore an accurate pose parameters estimation. The segmentation algorithm can be expressed as follows:

1. For a given prior shape, initialize pose parameters: $s(t=0)=1, \theta(t=0)=$ $0, \mu(t=0)=\mathbf{0}$ and build $\psi(\mathbf{x}, t=0)$. Initialize $\phi(\mathbf{x}, t=0)$ within a narrow band.

2. Evolve the active contour according to (16).

3. Re-initialize $\phi(\mathbf{x}, t)$ within a narrow band.

4. Retrieve and update pose parameters $(s(t), \theta(t), \mu(t))$ using the simplex. 
5. While the contour has not converged loop steps 2 to 5 . Otherwise trigger the shape constraint weight function increase $\left(t=t_{1}\right)$.

6. Repeat steps 2 to 4 until final convergence.

In our experiments a narrow band half size of three pixels yields satisfying results and re-initialization is achieved in using the fast scheme presented in [21. We adapted the latter method to preserve sub-pixel accuracy of the segmenting curve.

\subsection{Results and Discussion}

We show experimental results with three different kinds of $2 \mathrm{D}$ grey-level images. The first image depicts a manufactured toy extracted from the Amsterdam Library of Object Images (ALOI). The second and third apply to remote sensing and medical imaging respectively. Prior shapes used in experiments represent the exact boundaries of the object we aim at segmenting. In the case of remote sensing imagery, prior shape knowledge might come from cartographic data as we showed in [16. A perfect single prior shape is however not realistic in the case of medical imaging since shape variabilities of organs across patients, views and acquisition means compel to use statistical frameworks. Nevertheless, we performed experiments with the brain image in order to demonstrate the robustness our scheme on a wider range of images which exhibit a lack of discrimination of the target from the background. In the following experiments, the initial contour is similar to the shape prior and transformed by a similarity transformation which parameters are called $\xi_{i n i}=\left(s_{i n i}, \theta_{i n i}, \mu_{i n i}\right)$ in the rest of the paper. We propose to quantify and compare the performances of the simplex and gradient descent schemes in computing the absolute error between $\xi_{\text {ini }}$ and their estimates $\hat{\xi}_{\text {opt }}$ retrieved by each method (table 11). We refer the reader to 8 for implementation details about the gradient descent technique. As the shape priors perfectly match the objects to be segmented in the images, the quantitative results of table 1 also measure the accuracy of the segmentation achieved

Table 1. Absolute errors between the initial similarity transformation parameters $\xi_{\text {ini }}$ and their estimates $\hat{\xi}_{\text {opt }}$ by the simplex and gradient descent schemes

\begin{tabular}{|c|c|c|c|}
\hline & \multicolumn{2}{|c|}{ Absolute } & error \\
\hline Experiments & $\Delta \theta$ in degrees & $\Delta s$ & $\Delta \mu$ in pixels \\
\hline 1. Spatio-temporal $\lambda+$ simplex, fig. 2. $\mathrm{c}$ & -0.6 & 0.05 & $(-0.2,-0.05)$ \\
\hline 2. Spatio-temporal $\lambda+$ simplex, fig. 3.a & -0.1 & -0.01 & $(-0.04,-0.60)$ \\
\hline 3. Spatio-temporal $\lambda+$ simplex, fig. 3.b & 1.0 & 0.00 & $(0.65,-0.15)$ \\
\hline 4. Spatio-temporal $\lambda+$ simplex, fig. [3. $\mathrm{c}$ & -0.2 & 0.00 & $(0.25,-0.02)$ \\
\hline 5. $\lambda=$ cst + simplex, fig. 5 a & 0.9 & 0.05 & $(-0.12,-0.20)$ \\
\hline 6. $\lambda=c s t+$ grad. desc, fig. 5.b & 0.9 & 0.05 & $(-0.15,-0.20)$ \\
\hline 7. Spatio-temporal $\lambda+$ grad. desc, fig. [5]c & -4.0 & -0.08 & $(0.65,-3.30)$ \\
\hline 8. Spatio-temporal $\lambda+$ grad. desc, fig. [5] d & 122.6 & -0.20 & $(0.50,-3.70)$ \\
\hline
\end{tabular}


by the spatio-temporal weighting technique.The first experiment illustrates the efficiency of the proposed scheme with a simple case and $s_{i n i}=2, \theta_{\text {ini }}=\pi$, $\mu_{\text {ini }}=(10,-10)$ (fig. 2). We compare the results obtained with a constant prior shape weight $(\lambda=100, \lambda=10)$ and our scheme. The case $(\lambda=100)$ illustrates the lack of flexibility of the active contour because of a predominant shape constraint (fig. 2, a where $\lambda$ is intentionally high). The constraint prevents and penalizes the motion of the active contour which image-based force is not significant enough to segment accurately the border of the object. In that case the correct pose parameters can not be estimated and the contour finally reached a local minimum of the functional corresponding to an unsatisfying segmentation result. In the second case $(\lambda=10)$, the shape constraint is not strong enough to cope with the erasure which corrupts the object in the image (fig. 2.b), which also corresponds to a local minimum of the energy. Finally the spatio-temporal scheme shows how a low and spatially constrained shape prior weight enables to reach a rough segmentation which is at the end improved when the constraint is reinforced. The resulting segmentation is satisfying (fig. 2 c) and the pose parameters are estimated with a good accuracy (table 1, row 1). The second set of experiments is performed with images which exhibit lack of discrimination of the target object from the background and the proposed spatio-temporal scheme (fig. 3). In the first case the toy pattern was flipped, resized and duplicated to make a background with similar local radiometric properties to the target. In the first experiment (fig. 3. a), the initial contour is transformed according to $s_{i n i}=0.75, \theta_{i n i}=\pi / 4, \mu_{i n i}=(10,-10)$. We can see that peripheral patterns

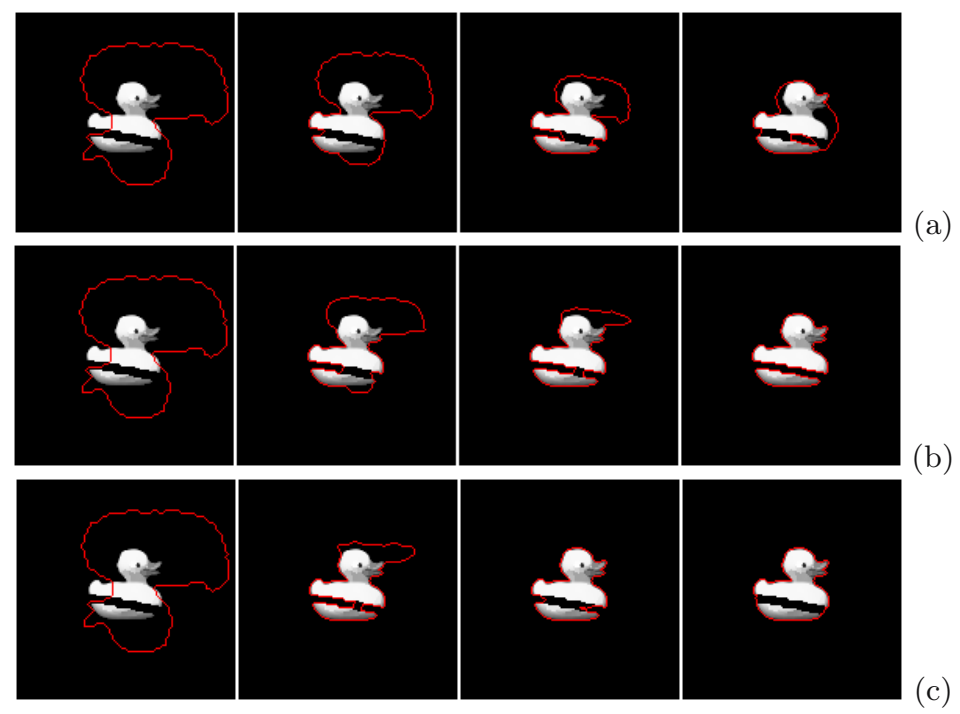

Fig. 2. Segmenting curve evolution from left to right: comparison between constant and spatio-temporal shape prior weight. (a) $\lambda=100$, (b) $\lambda=10$ (c) spatio-temporal function with $d_{0}=10, \lambda_{\min }=20$ and $\lambda_{\max }=100$. 

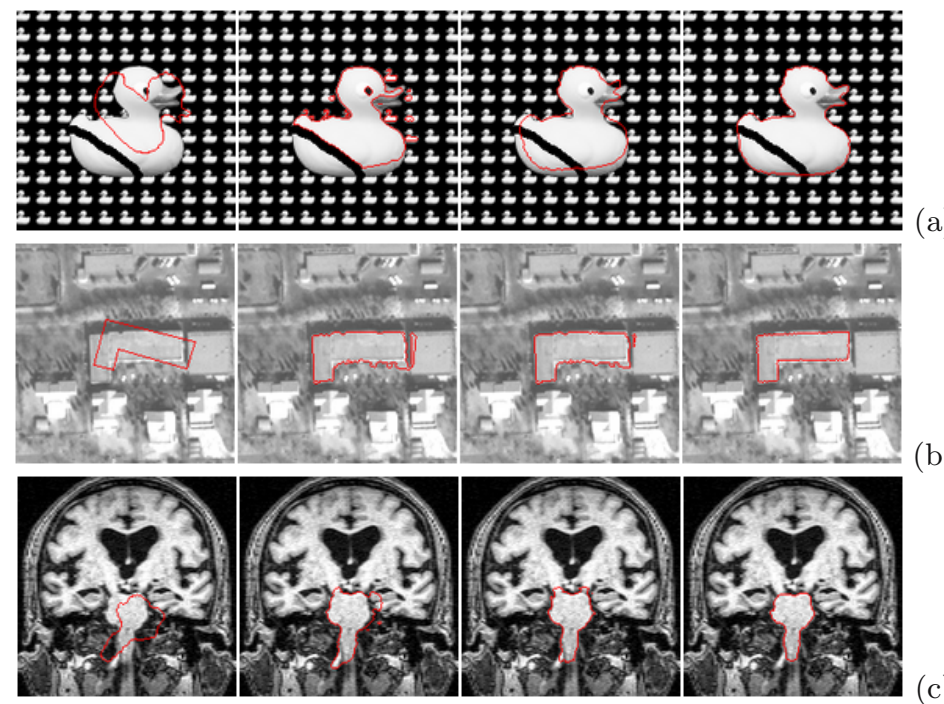

Fig. 3. Segmenting curve evolution (from left to right) with spatio-temporal shape prior weight function and lack of discrimination of the target from the background. (a) $d_{0}=15, \lambda_{\min }=15$ and $\lambda_{\max }=100$ (b) $d_{0}=7, \lambda_{\min }=27$ and $\lambda_{\max }=70$ (c) $d_{0}=10$, $\lambda_{\min }=50$ and $\lambda_{\max }=100$.

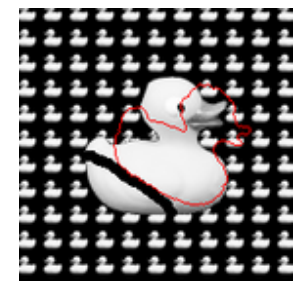

(a)

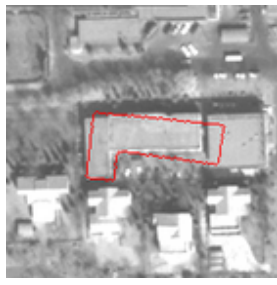

(b)

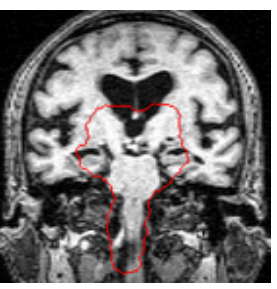

(c)

Fig. 4. Failed segmentations with constant shape prior weight and lack of discrimination from background. (a) $\lambda=20$ (b) $\lambda=50$ (c) $\lambda=70$.

similar to the target do not affect the final segmentation result. Indeed, during the convergence process the prior shape is flexible enough to allow segmentation of the patterns and topology changes. At the end of the process, the target is correctly segmented as the erasure artifact is overcome by constraint enhancement. The effect of the freedom space is visible in figure 3 a as small toy patterns are segmented within a neighborhood of the main segmenting curve. The figure 4. a shows the unsuccessful segmentation result obtained with a constant shape constraint $(\lambda=20)$. The over-estimated shape weight restricts the motion of the active contour which is trapped by a local minimum. The second case ap- 
plies to a remote sensing image depicting two buildings with similar radiometric properties (fig. 3. b, fig. 4.b). In this case we expect to segment the L-shaped building in the center of the image. The initial contour is transformed according to $s_{i n i}=1.0, \theta_{i n i}=0.3, \mu_{i n i}=(7,-5)$. The segmentation process in figure 3. $\mathrm{b}$ shows how the spatio-temporal scheme conveys flexibility to the segmenting curve while avoiding to segment the squared building nearby the target. A hard and constant shape prior incorporation yields the unsuccessful segmentation of parts of both buildings (fig. (4) b). The main reason of such result is the inability of the segmenting curve to split at the border between the two buildings because of a two high shape constraint. As a result, a part of the second building is also segmented as it shares similar image statistical properties to the target. Finally the ability of the segmenting curve to undergo topological changes is yet illustrated with the medical image in figure 3. $\mathrm{c}\left(s_{i n i}=1.1, \theta_{i n i}=0.7, \mu_{i n i}=(3,3)\right)$. The spatially constrained active contour prevents from spreading all over the image while retaining local flexibility within the freedom space and yields the correct solution opposed to the constant shape constraint (fig. [4. $\mathrm{c}$ ). The quantitative results of these three cases (table1, rows 2 to 4 ) validate the performances of the simplex algorithm as well as our spatio-temporal scheme. Indeed, the absolute error between the initial transform and its estimate is less than one degree for the angle, less than one pixel for the translation and less than 0.05 for the scale factor. Results displayed in figure 5 compare the simplex and gradient descent schemes for the estimation of the similarity transformation parameters. The two first experiments carried out with a simple case $\left(s_{i n i}=1.5, \theta_{i n i}=\pi / 4\right.$, $\left.\mu_{i n i}=(2,0)\right)$ show comparable good results with a constant prior shape weight, which is confirmed by the parameter estimate errors (table1, rows 5-6). However the two last results are wrong segmentations with the spatio-temporal schemes and the gradient descent (fig. 5. c-d) whereas they were successful with the simplex algorithm (fig. 3 a, fig. 2. c). Sensitivity to local minima of the gradient descent scheme might explain these incorrect segmentations. The comparison of the parameters estimations (table 1 rows $2 \& 7$ and $1 \& 8$ ) also demonstrates that the simplex outperforms the gradient descent in these cases. In the end, we com-

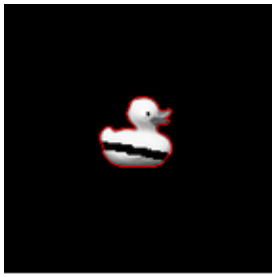

(a)

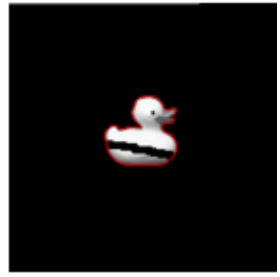

(b)

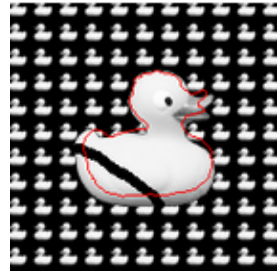

(c)

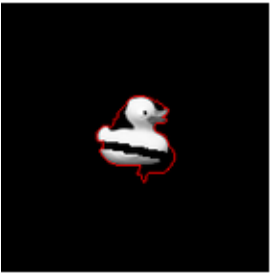

(d)

Fig. 5. Segmentation results comparison with the simplex and the gradient descent schemes. (a-b) successful results with $\lambda=75$ : (a) simplex method (b) gradient descent. (c-d) unsuccessful results of the grad. desc method with the spatio-temporal scheme: (c) $d_{0}=15, \lambda_{\min }=15$ and $\lambda_{\max }=100$ (d) $d_{0}=10, \lambda_{\min }=20$ and $\lambda_{\max }=100$. 


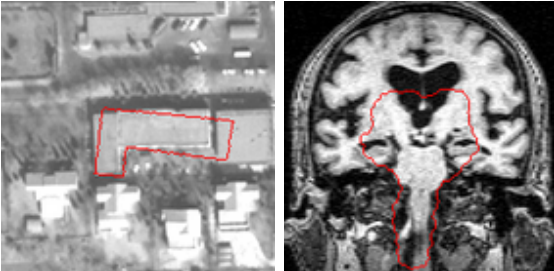

(a) (b)

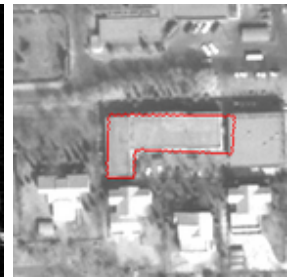

(c)

Fig. 6. Failed segmentations with: (a) sole temporal variation of the shape prior weight amplitude between $\lambda_{\min }=30$ and $\lambda_{\max }=70$ (no spatial relaxation). (b) spatiotemporal model with ramp-like spatial profile. (c) spatio-temporal model with no time transition (Heaviside step function for $\lambda_{a}(t)$ and $\left.d(t)\right)$.

pare our spatio-temporal shape constraint scheme exposed in (8) with simpler temporal and spatial profiles functions. In figure [6] a is displayed the segmentation result with a sole temporal weight variation (no spatial relaxation) using the same $\left(\lambda_{\min }, \lambda_{\max }\right)$ values as the experiment carried out in figure 3, b. This result is unsuccessful unlike the spatio-temporal case. With the purely temporal method, the low shape constraint applied at the beginning of the convergence process does not allow to overcome image artifacts because of the lack of spatial flexibility. As a consequence, the segmenting curve diverges from the target and biases pose parameters estimation. When the shape constraint is reinforced at the end, the curve is far from the target with a significant shape weight, which makes the segmentation process very sensitive to local minima and explain the erroneous result. The second experiment similar to the one in figure (3. $\mathrm{c}$ ) was carried out with the proposed spatio-temporal technique but with a different spatial profile. Instead of the exponential formulation, a simpler piecewise linear function was used: $\lambda_{\text {space }}(\psi(\mathbf{x}))=|\psi(\mathbf{x})| /(2 d(t))$ if $|\psi(\mathbf{x})|<2 d(t)$, else $\lambda_{\text {space }}(\psi(\mathbf{x}))=1$. With this more intuitive ramp-like spatial profile, the result is incorrect (fig. 6, b). Such profile reinforces too much the weight in a very close neighborhood of the prior shape. As a result, the segmenting curve is not flexible enough to roughly capture the object in the image and prevents from a good result when the shape constraint is enhanced. Attempts with a larger freedom space to cope with this drawback would not yield better results as the shape constraint would be too loose in space. The proposed spatial profile in (8) alleviates this problem since it enables a low weight close to the reference template while retaining a strong constraint farther. Finally, we repeat the experiment of figure $3 \mathrm{~b}$ b while replacing the temporal profiles $\lambda_{a}(t)$ and $d(t)$ by two Heaviside step functions. The shape constraint is then suddenly enhanced instead of progressively. The unsuccessful result of figure [6] shows that the spatio-temporal scheme proposed originally performs better. Before shape constraint reinforcement, the pose parameters estimation can be slightly biased as the contour roughly segments the object in the image. A progressive reduction of the freedom space and increase of the constraint amplitude is therefore needed 
for the parameters estimation scheme to catch up with the evolving curve which converges to the target because of its gradually enhanced regularization. Such adjusting procedure is not possible with a sudden constraint application as the active contour directly embodies the prior shape with inaccurate pose parameters. As a consequence, the evolving curve might be far from the target with a high and uniform shape constraint, which makes it sensitive to local minima and prevents from satisfying segmentation (fig. [6]c).

\section{Conclusion}

We have presented a soften single shape prior incorporation for model-based level set segmentation of objects from corrupted data in variational frameworks. The proposed scheme is intended to circumvent the difficult tuning of the weight balancing shape and image information. Most of the time this constant parameter is under or over-estimated, which yields unsuccessful segmentation results corresponding to local minima of the energy functional. The proposed shape constraint is spatially relaxed in a neighborhood of the prior shape to overcome the issue of local minima. The constraint is later enhanced to overcome image data corruptions. Experiments carried out with different kinds of images demonstrated the efficiency of our spatio-temporal approach compared to the shape prior integration achieved by a constant constraint. We also empirically demonstrated the good performances of our approach with respect to simpler temporal and spatial profile functions. Future works may be dedicated to the building up of a theoretical basis enabling to retrieve such profile functions from the image and shape prior information instead of the exposed ad hoc definitions. Besides, we have proposed an alternative to the gradient descent scheme for pose parameters estimation by using the downhill simplex method. The simplex algorithm is easier to tune and estimates more robustly pose parameters than the gradient descent scheme. Practically more tractable, the proposed method is simple, easy to implement and can be applied to any segmentation problem incorporating single prior shape constraints.

\section{References}

1. Staib, L.H., Duncan, J.S.: Boundary finding with parametrically deformable models. IEEE Transactions on Pattern Analysis and Machine Intelligence 14 (1992) 1061-1075

2. Cootes, T., Cooper, D., Taylor, C., Graham, J.: Active shape models - their training and application. Computer Vision and Image Understanding. 61 (1995) 38-59

3. Leventon, M., Grimson, E., Faugeras., O.: Statistical shape influence in geodesic active contours. Comp. Vision and Patt. Recon. (CVPR) (2000)

4. Chen, Y., Tagare, H., Thiruvenkadam, S., Huang, F., Wilson, D., Gopinath, K., Briggs, R., Geiser, E.: Using prior shapes in geometric active contours in a variational framework. International Journal of Computer Vision 50(3): 315-328 (2002)

5. Cremers, D., Tischhauser, F., Weickert, J., Schnorr, C.: Diffusion snakes : introducing statistical shape knowledge into the mumford-shah functional. ICCV (2002) 
6. Paragios, N., Rousson, M.: Shape priors for level set representations. European Conference in Computer Vision 2 (2002) 78-92

7. Foulonneau, A., Charbonnier, P., Heitz, F.: Geometric shape priors for regionbased active contours. IEEE Int. Conf. Image Processing, ICIP 2003 (2003)

8. Chan, T., Zhu, W.: Level set based shape prior segmentation. Technical report, UCLA (2003)

9. Cremers, D., Sochen, N., Schnörr, C.: Towards recognition-based variational segmentation using shape priors and dynamic labeling. Intl. Conf. on Scale-Space Theories in Computer Vision (2003)

10. Cremers, D., Soatto, S.: A pseudo-distance for shape priors in level set segmentation. Proc. ICCV'2003 (2003)

11. Cremers, D., Osher, S., Soatto, S.: Kernel density estimation and intrinsic alignment for knowledge-driven segmentation: teaching level sets to walk. DAGM'04: 26th Pattern Recognition Symposium (2004)

12. Tsai, A., Yezzi, A., Wells, W., Tempany, C., Tucker, D., Fan, A., Grimson, E., Willsky, A.: Model-based curve evolution technique for image segmentation. IEEE Conference on Computer Vision and Pattern Recognition 1 (2001) 463-468

13. Kass, M., Witkin, A., Terzopoulos, D.: Snakes : active contour models. 1st International Conference on Computer Vision, pp. 259-268 (1987)

14. Osher, S., Sethian, J.: Fronts propagating with curvature-dependent speed: algorithms based on Hamilton-Jacobi formulations. Journal of Computational Physics, 79, pp. 12-49 (1988)

15. Sethian, J.: Level set methods and fast marching methods: evolving interfaces in computational geometry, fluid mechanics, computer vision and materials science. Cambridge University Press (1999)

16. Bailloeul, T., Prinet, V., Serra, B., Marthon, P., Chen, P., Zhang, H.: Digital building map refinement from knowledge-driven active contours and very high resolution optical imagery. Proc. ISPRS Hannover Workshop (2005)

17. Chen, Y., Thiruvenkadam, S., Tagare, H., Huang, F., Wilson, D., Geiser, E.A.: On the incorporation of shape priors into geometric active contours. IEEE 1st Worshop on Vatiational Framework and Level Sets methods (2001)

18. Nelder, J., Mead, R.: A simplex method for function minimization. Computer Journal 7 (1965) 308-313

19. Paragios, N., Deriche, R.: Geodesic active regions: A new paradigm to deal with frame partition problems in computer vision. Journal of Visual Communication and Image Representation, 13 (2002) 249-268

20. Paragios, N., Rousson, M., Ramesh, V.: Matching distance functions: A shape-toarea variational approach for global-to-local registration. European Conference in Computer Vision (2002)

21. Yui, S., Hara, K., Zha, H., Hasegawa, T.: A fast narrow band method and its application in topology-adaptative 3-d modeling. 16th Intl Conf. on Pattern Recognition (ICPR'02) Vol. 4 (2002) 